\title{
Method for quantitative estimation of thermal thresholds in patients
}

\author{
H. FRUHSTORFER, U. LINDBLOM, AND W. G. SCHMIDT \\ From the Institute of Physiology, University of Marburg, Marburg/Lahn, GFR, and \\ Department of Neurology, Karolinska Institute, Huddinge Hospital, Huddinge, Sweden
}

SYNOPSIS A quantitative method for the examination of thermal sensibility was applied in 26 normal subjects and in patients with various neurological disorders. The stimulation technique resembled Békésy audiometry: the patient reversed the direction of the temperature change of a thermode whenever warm, cold, or thermal pain thresholds were reached. The resulting temperature curve enables a quantitative description of the subject's thermal sensibility and of the degree of impairment displayed by neurological patients.

It is well known that a disturbance of the temperature sense occurs in many neurological patients as a consequence of lesions in the peripheral or central nervous system. It usually occurs together with a disturbance of other sensory modalities such as cutaneous pain, but it may also appear separately (Goldscheider, 1926).

For a closer study of changes in thermal sensibility, a quantitative technique is necessary which allows reliable measurement of warm, cold, and thermal pain thresholds by pure thermal stimuli without tactile components. The method should be easy and quick enough to be combined with routine neurological examination. Although in neurophysiological and psychophysical research several methods have been developed for the study of temperature sensibility (for references $c f$ Kenshalo, 1970), all of them are too complicated and time consuming to be applied clinically. The technique described here has proved to be easy enough to be used routinely and repeatedly in patients in whom it was desirable to establish the degree or the temporal course of a neurological disorder affecting thermosensibility.

\section{METHODS}

\section{STIMULATOR}

The thermostimulator has a rectangular stimulating surface of $25 \times 50 \mathrm{~mm}$ (Fig. 1), and it operates on the Peltier principle. It consists of semiconductor junc-

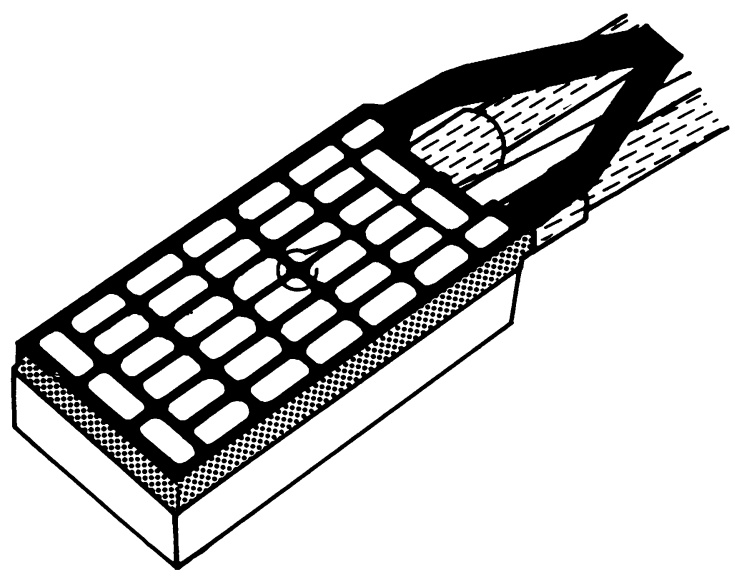

FIG. 1 The Marstock stimulator. A thermocouple is fixed to the centre of the stimulating surface (area $25 \times 50 \mathrm{~mm})$.

tions which produce a temperature difference between upper and lower side of the stimulator when a current is passed through them. As the reverse side of the stimulator is thermally buffered by a metal block perfused with water of $30^{\circ} \mathrm{C}$ temperature, the stimulating surface can be either warmed or cooled depending on the direction of the current. The temperature is measured by a thermocouple and recorded by a pen recorder. 


\section{PROCEDURE}

The stimulator is applied to the skin of the patient and a constant current source is connected. The patient is instructed to reverse a switch as soon as he perceives the stimulator getting warm. Reversing the switch changes the direction of the current and, as a consequence, the stimulating surface is cooled. The patient has to reverse the switch again as soon as he perceives the stimulator becoming cold. This procedure is continued for one or two minutes while the temperature between stimulator and skin is recorded.

After warm and cold thresholds have been obtained, cold pain and heat pain thresholds are determined with a slightly different procedure. The patient is instructed to activate the switch when he perceives the stimulus as painful. As soon as the stimulator has reached again an indifferent temperature of about $30^{\circ} \mathrm{C}$, the current is reversed by the operator and the next pain stimulus is applied.

In routine work, temperature change rates from $1.0^{\circ} \mathrm{C} . \mathrm{s}^{-1}$ to $1.5^{\circ} \mathrm{C} . \mathrm{s}^{-1}$ have been shown to be suitable for most skin areas. For this rate the stimulator needs a current of approximately 3.0A. When very sensitive areas are being examined, slower rates should be preferred in order to avoid frequent switching. If a constant current source is used, the rate of temperature change remains fairly constant over the range between $20^{\circ} \mathrm{C}$ and $50^{\circ} \mathrm{C}$.

Because of the collaboration between Marburg and Stockholm the method was named 'Marstock'.

\section{RESULTS}

The quantitative method for the examination of thermal sensibility (Marstock method) has been applied in 26 normal subjects and in about 100 neurological patients with different peripheral or central neurological disorders. All subjects and patients, even if they were old or under the influence of sedatives, were able to perform this test. Usually it took three to five minutes to obtain cold, warm, and thermal pain thresholds from one skin area. So far only patients with uraemic polyneuropathy have been studied systematically, the result of which will be described separately (Fruhstorfer et al., 1976).

An illustrative record from the hand of a normal subject is shown in Fig. 2. In most recordings an initial rise of cold and warm thresholds to higher temperatures occurs. Usually constant values are obtained within a minute. These stable thresholds are measured, and the difference between them-the warm-cold difference limen--is calculated. It roughly represents the indifferent temperature range in which no thermal sensations occur. After these values have been obtained, cold pain and heat pain thresholds are

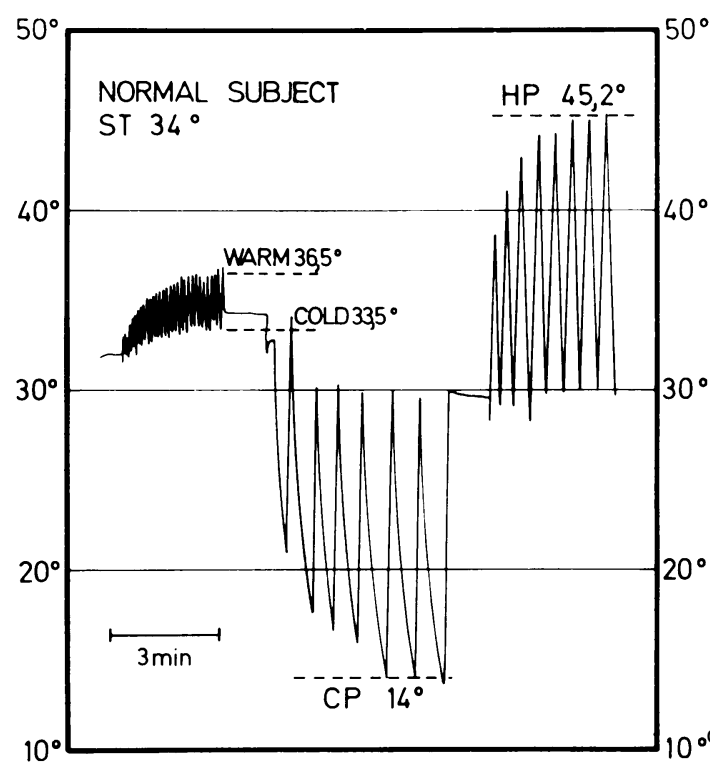

FIG. 2 Typical Marstock record from the right thenar region of a normal subject. When determining thermal pain thresholds the current was reversed at indifferent temperatures on the operator's request. (ST=skin $\frac{\rho}{8}$ temperature. $C P=$ cold pain threshold, $H P=$ heat pain $\rightarrow$ threshold).

determined. Whereas heat pain thresholds could be examined in all patients, cold pain thresholds seem to depend considerably on other variables, such as reporting criteria, and therefore could not be measured in all subjects. These pain thresholds also increase initially, as is clearly seen in Fig. 2, before a steady level is reached.

If thermal thresholds are determined at various body sites, different values are obtained, reflecting differences in thermal sensibility (Fig. 3). The threshold values from the right cheek, hand (thenar region), abdomen $\left(\mathrm{T}_{9}\right.$ dermatome), and lateral foot (sural nerve) of 26 normal subjects show that the interindividual variability is considerable (Table). The intra-individual variability is, however, much smaller, as preliminary measurements have shown. A comparison of these normal data with the values obtained in patients with uraemic neuropathy indicates that the warm-cold difference limen is a sensitive parameter to distinguish between normal and pathological thermosensibility (Fruhstorfer et al. 1976). Figure 4 shows abnormal thermal thresholds which were found in an 18 year old girl suffering from a subacute polyneuropathy of unknown cause, manifesting itself by distal 
Face $(N \geq 2)$

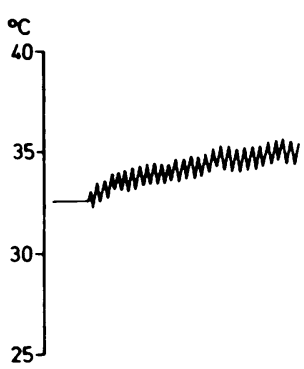

Th 9

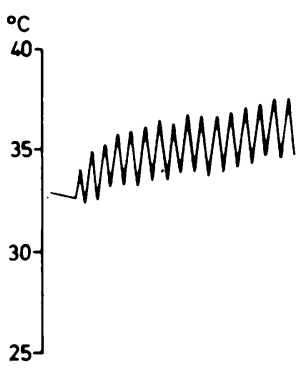

Hand ( $N$ medianus)

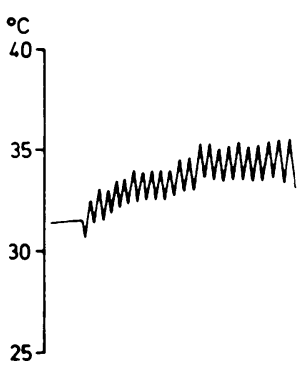

Foot ( $N$ suralis)

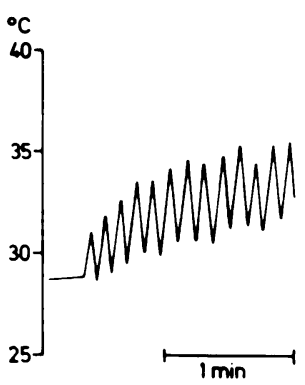

FIG. 3 Marstock records from the face, hand, abdomen, and foot of a normal subject.

paresis, diminished or abolished deep reflexes, and a marked increase in vibration thresholds, specially in the right foot and leg. Whereas the recordings from both hands were within normal limits, warm-cold difference limen and thermal pain thresholds were increased on the right foot. On the left foot the heat pain threshold was unusually low, indicating hyperalgesia.

In cases of unilateral peripheral or central neurological disorders affecting temperature sense, side differences in Marstock values give a clear indication of the degree of the sensory defect. In a male student (20 years old), five years after right median nerve suture, warm-cold difference limen, together with vibratory and tactile thresholds, are still raised, demonstrating incomplete regeneration (Fig. 5).

In a female patient ( 28 years old) afflicted with multiple sclerosis, the most conspicuous finding in the Marstock recordings (Fig. 6) were the variable thermal thresholds and the abnormally high warm-cold difference limen on the left foot compared with normal values on the right foot. This unilateral disturbance of thermosensibility was accompanied by high vibratory and tactile thresholds in the right foot and leg. The findings suggest a lesion located on the right side of the thoracic cord.

\section{DISCUSSION}

The Marstock method has proved to be easy, reliable, and fast in estimating quantitatively warm, cold, and thermal pain thresholds in patients. It resembles threshold determination in a Békésy audiometer and has the advantage that the operator is not directly involved in the measuring procedure, whereas the patient plays the active part. A similar method has also been used by Kenshalo and Scott (1966) when studying the temporal course of thermal adaptation.

The method provides relatively pure thermal stimuli which should be given preference over the placing of objects of different temperature on the skin, because a tactile cue may enable the patient to guess the temperature of the object on the basis of cold sensitivity of slowly adapting mechanoreceptors (for details see Hensel, 1973).

The finding that the thresholds determined by the Marstock technique are higher than those obtained by Kenshalo (1970) with a different method can be explained by the dependence of the Marstock values on the skin-brain information transmission time. As conduction velocity is low in the afferent thermal

TABLE

COLD THRESHOLD, WARM THRESHOLD, AND WARM-COLD DIFFERENCE LIMEN MEASURED AT DIFFERENT SKIN AREAS IN 26 NORMAL SUBJECTS (STUDENTS AND LABORATORY WORKERS, AGE RANGE 18-39 YEARS)

\begin{tabular}{|c|c|c|c|c|c|c|c|c|c|c|c|c|}
\hline & \multicolumn{3}{|c|}{ Face $\left(N . V_{2}\right)$} & \multicolumn{3}{|c|}{ Hand (thenar) } & \multicolumn{3}{|c|}{ Abdomen $\left(T_{9}\right)$} & \multicolumn{3}{|c|}{ Foot $(N$. suralis $)$} \\
\hline & 4 & 50 & 96 & 4 & 50 & 96 & 4 & 50 & 96 & 4 & 50 & 96 \\
\hline Cold & 33.0 & 34.5 & 37.0 & 30.5 & 33.0 & 35.0 & 30.5 & 34.0 & 38.0 & 30.5 & 33.0 & 35.0 \\
\hline Warm & 33.5 & 35.5 & 38.5 & 32.0 & 35.0 & 38.0 & 34.5 & 36.5 & 39.5 & 34.5 & 36.5 & 41.5 \\
\hline Diff. & 0.5 & 1.0 & 2.5 & 0.5 & 2.0 & 4.0 & 1.0 & 2.5 & 5.0 & 2.0 & 3.0 & 7.0 \\
\hline
\end{tabular}

Four, 50 and 96 percentiles are given. All values in ${ }^{\circ} \mathrm{C}$. 


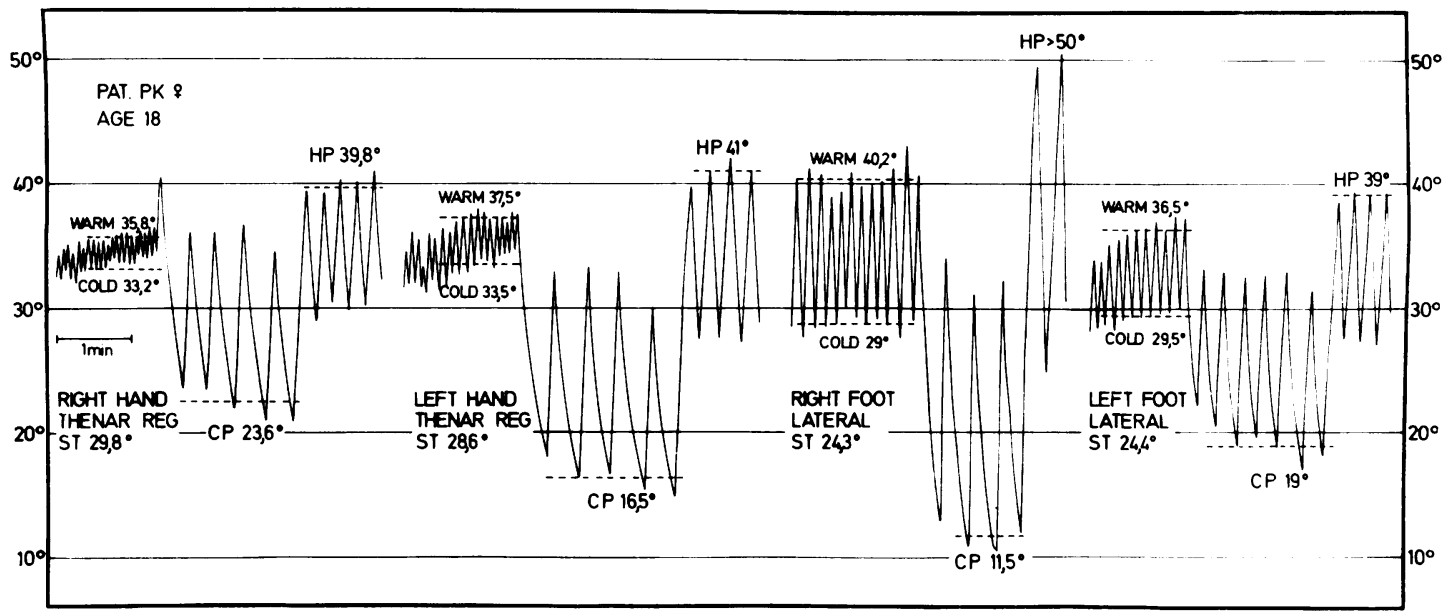

FIG. 4 Record from both hands and feet of a patient with peripheral neuropathy. On the right foot the warm-cold difference limen was great and the heat pain threshold was not reached (the current was reversed at $50^{\circ} \mathrm{C}$ on the operator's request in order to avoid burning). The heat pain threshold on the right foot is very low.

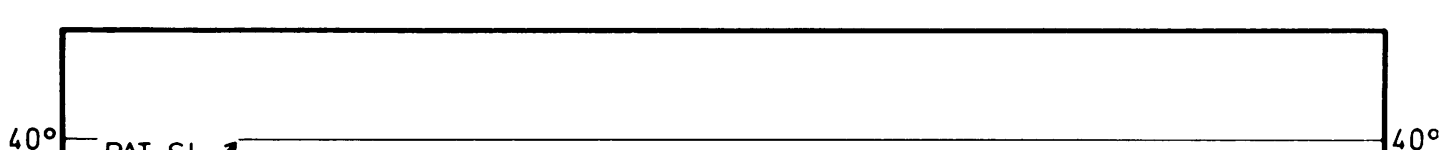

AGE 20

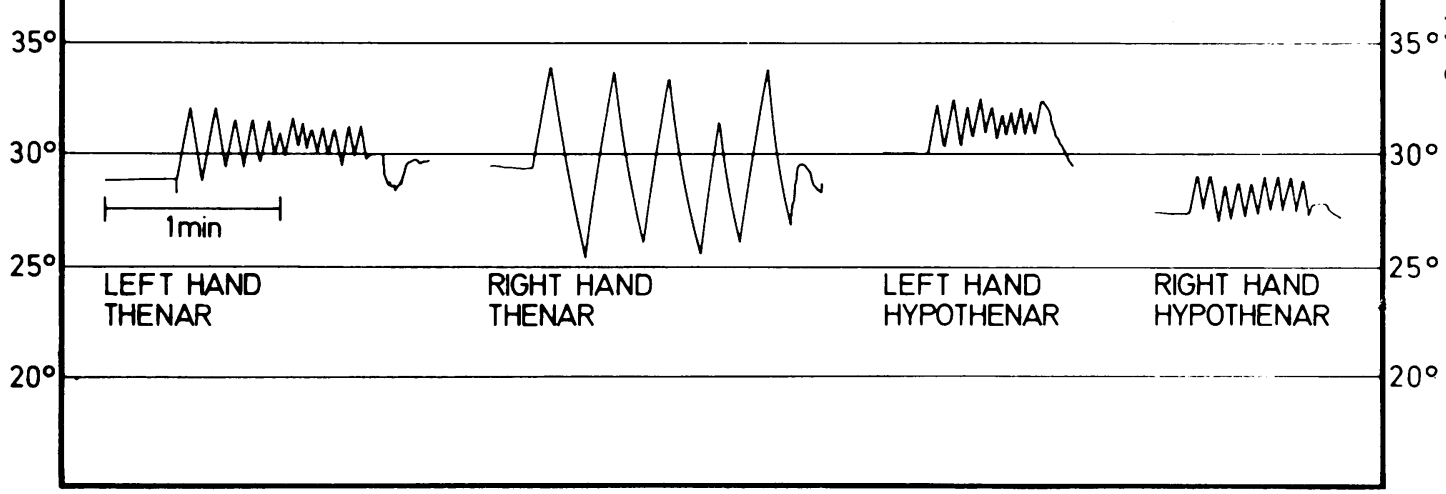

FIG. 5 Record from both hands of a patient five years after right median nerve suture. Warm-cold difference limen is greater on the right thenar.

pathways of man (Fruhstorfer, 1976) the deviation of the Marstock thresholds from the absolute thresholds (reported by Kenshalo) increases if the temperature change rate increases beyond $0.5^{\circ} \mathrm{C} . \mathrm{s}^{-1}$ and it is greater with a longer afferent pathway. Pathological slowing of reaction time due to slow central information processing which may appear in old patients, disorders of the central nervous system, or under the influence of drugs will also affect the threshold measurement. This error must be taken into account if increased values are found at all skin areas of a patient.

In the present procedure warm and cold thresholds were determined first, followed by the determination 


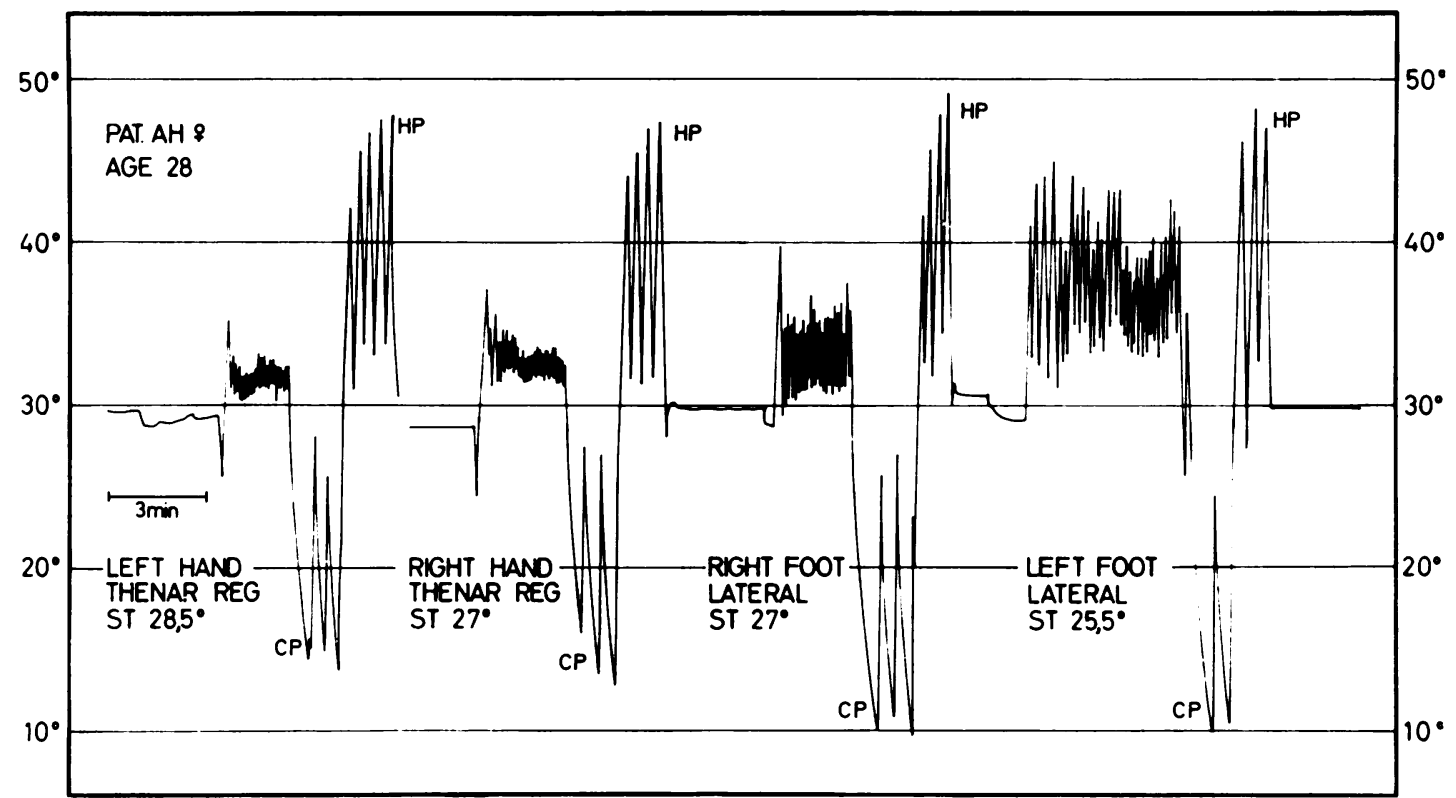

FIG. 6 Record from both hands and feet of a patient with multiple sclerosis. Great warm-cold difference limen with variable thresholds on the left foot.

of cold pain threshold and lastly of the heat pain threshold. This sequence was selected because heat stimulation may sensitise polymodal nociceptors (King et al., 1976) and perhaps influence the excitability of the warm and cold receptors.

Although the present thermode is suitable for most skin areas, a smaller thermode should be preferred in places like the face or the finger tip, where the large thermode does not make good contact with all of its stimulating surface. A well-defined size of the stimulated areas is important when determining thermal thresholds (Hensel, 1952; Kenshalo, 1970).

Although the Marstock method does not give the exact values of thermal thresholds, it is so far the only method by which a fast, quantitative, and reproducible estimation of thermal thresholds is possible. It may be a sensitive index of manifest or subclinical neuropathies affecting unmyelinated and thin myelinated afferent axons, or the spinothalamic pathway.

We are grateful to Ingolf Detering who built the thermodes. The work was supported by the Deutsche Forschungsgemeinschaft ( Fr 265) and Karolinska Institute Fonder.

\section{REFERENCES}

Fruhstorfer, H. (1976). Conduction in the afferent thermal pathways of man studied by cortical evoked responses, reaction time and regional anaesthesia. In Sensory
Functions of the Skin in Primates. Edited by Y. Zotterman. Pergamon Press: Oxford. pp. 355-365.

Fruhstorfer, H., Goldberg, J. M., Lindblom, U., and Schmidt, W. G. (1976). Temperature sensitivity and pain thresholds in patients with chronic uremia and peripheral neuropathy. In Sensory Functions of the Skin in Primates. Edited by Y. Zotterman. Pergamon Press: Oxford. pp. 507-517.

Goldscheider, A. (1926). Pathologie des Temperatursinns. In Handbuch der normalen und pathologischen Physiologie, vol. 11, pp. 161-164. Edited by O. Bethe, G. Bergmann, G. Embden, and A. Ellinger. Springer: Berlin.

Hensel, H. (1952). Physiologie der Thermoreception. Ergebnisse der Physiologie, 47, 166-368.

Hensel, H. (1973). Cutaneous thermoreceptors. In Handbook of Sensory Physiology, vol. 2. Edited by A. Iggo. Springer: New York.

Kenshalo, D. R. (1970). Psychophysical studies of temperature sensitivity. In Contributions to Sensory Physiology, vol. 4, pp. 17-74. Edited by W. D. Neff. Academic Press: New York.

Kenshalo, D. R., and Scott, H. A. (1966). Temporal course of thermal adaptation. Science, 151, 1095-1096.

King, J. S., Gallant, P., Myerson, V., and Perl, E. R. (1976). The effect of anti-inflammatory agents on the responses and the sensitization of unmyelinated (C) fibre polymodal nociceptors. In Sensory Functions of the Skin in Primates, pp. 441-454. Edited by Y. Zotterman. Pergamon Press: Oxford. 